\title{
CPB-3 and CGH-1 localize to motile particles within dendrites in C. elegans PVD sensory neurons
}

\author{
Kathrin Spendier ${ }^{1 *}$, Eugenia C. Olesnicky ${ }^{2 *}$, Daniel Forand ${ }^{2}$, Margaret Wolf ${ }^{3}$ and Darrell J. Killian ${ }^{3^{*}}$ (D)
}

\begin{abstract}
Objective: RNA-binding proteins (RBPs) are important regulators of gene expression that influence mRNA splicing, stability, localization, transport, and translational control. In particular, RBPs play an important role in neurons, which have a complex morphology. Previously, we showed that there are many RBPs that play a conserved role in dendrite development in Drosophila dendritic arborization neurons and Caenorhabditis elegans (C. elegans) PVD neurons including the cytoplasmic polyadenylation element binding proteins (CPEBs), Orb in Drosophila and CPB-3 in C. elegans, and the DEAD box RNA helicases, Me31B in Drosophila and CGH-1 in C. elegans. During these studies, we observed that fluorescently-labeled CPB-3 and CGH-1 localize to cytoplasmic particles that are motile, and our research aims to further characterize these RBP-containing particles in live neurons.

Results: Here we extend on previous work to show that CPB-3 and CGH-1 localize to motile particles within dendrites that move at a speed consistent with microtubule-based transport. This is consistent with a model in which CPB-3 and CGH-1 influence dendrite development through the transport and localization of their mRNA targets. Moreover, CPB-3 and CGH-1 rarely localize to the same particles suggesting that these RBPs function in discrete ribonucleoprotein particles (RNPs) that may regulate distinct mRNAs.
\end{abstract}

Keywords: RNA-binding proteins (RBPs), CPB-3, CGH-1, Ribonucleoprotein particles (RNPs), Posttranscriptional regulation, Caenorhabditis elegans, Dendrites, Total internal reflection fluorescence (TIRF) microscopy

\section{Introduction}

Dendrites are neuronal processes that are often highly branched such that they create a large sensory field or can receive synaptic information from many other cells. The proper development and maintenance of dendritic branches is important for sensory perception, learning, memory, and behavior, and defects in dendrite

\footnotetext{
*Correspondence: kspendie@uccs.edu; eolesnic@uccs.edu; dkillian@coloradocollege.edu

1 Physics Department and Center for the Biofrontiers Institute, University

of Colorado Colorado Springs, Colorado Springs, CO 80918, USA

${ }^{2}$ Department of Biology, University of Colorado Colorado Springs,

Colorado Springs, CO 80918, USA

${ }^{3}$ Department of Molecular Biology, Colorado College, Colorado Springs,

CO 80903, USA
}

development are associated with several neurological disorders [1, 2]. Significant evidence supports the view that RNA-binding proteins (RBPs) are important for dendritic morphology across species because they can regulate several posttranscriptional mechanisms such as mRNA transport, localization, and localized translation [3, 4].

Previously, we identified a suite of conserved RBPs that play a role in regulating dendrite development in Drosophila larval dendrite arborization (da) neurons and $C$. elegans PVD neurons [5-10]. Among these are Drosophila Orb and C. elegans CPB-3, which are cytoplasmic polyadenylation element binding proteins (CPEBs). CPEBs have been implicated in the regulation of mRNA translation by controlling poly-(A) tail length and interacting with the translation initiation complex $[11,12]$. Our 
previous work showed that $\mathrm{CPB}-3$ localizes to particles within the soma, dendrites, and axons of PVD neurons in C. elegans [6]. In addition, studies in vertebrates have implicated CPEB in mRNA transport and localization within dendrites [13-15].

Drosophila Me31B and C. elegans $\mathrm{CGH}-1$ have also been implicated in dendrite development and axon morphogenesis $[6,16,17]$. These RBPs are DEAD box RNA helicases related to $S$. cerevisiae Dhh1 and mammalian DDX6/RCK; broadly, DEAD box RNA helicases of this family localize to cytoplasmic ribonucleoprotein particles (RNPs), such as processing bodies (P-bodies), and have been implicated as regulators of mRNA translation and decay [18-21]. In C. elegans, CGH-1 localizes to particles in the soma and neurites of PVD neurons and colocalizes with mRNA decay factors within C. elegans touch receptor neurons where it plays a role in regulating axon regeneration and maintaining axon integrity $[6,17]$. In Drosophila da neurons, Me31B is localized in dendrites suggesting a possible role in mRNA transport or localization, or in regulating the translation, storage, and decay of mRNAs during transit and in localized RNPs [22].

To learn more about the molecular mechanisms by which CPB-3 and CGH-1 regulate dendrite development, we expressed and observed functional fluorescent fusion proteins within PVD neurons in live C. elegans. We find that $\mathrm{CPB}-3$ and $\mathrm{CGH}-1$ localize to particles within dendrites that undergo directional movement at a speed consistent with microtubule-based active transport. A much larger proportion of $\mathrm{CGH}-1$ particles displayed directed movement compared to CPB-3 particles. Of the particles exhibiting directed movement, $\mathrm{CPB}-3$ particles are more likely to exhibit anterograde movement away from the soma while CGH-1 particles often move bidirectionally within dendrites. In dual labeling experiments we find that CPB-3 and CGH-1 rarely label the same particles suggesting that these RBPs may regulate different mRNAs and/or function independently of each other. Together, our results are consistent with a model in which $\mathrm{CPB}-3$ and $\mathrm{CGH}-1$ regulate mRNAs during their transport within dendrites, perhaps playing a critical role in regulating dendrite morphogenesis.

\section{Main text}

\section{Results}

CPB-3 and CGH-1 localize to motile particles in PVD dendrites We previously identified CPB-3 and CGH-1 as RBPs that are important for dendrite morphogenesis of PVD neurons in C. elegans. Using RBP::GFP (green fluorescent protein) fusion proteins expressed in PVD neurons, we found that these RBPs localize to particles within the dendrites and soma, and we showed that these fusion proteins are functional because they rescue (albeit partially for $\mathrm{CPB}-3$ ) the dendrite defects in mutants [6]. To better characterize the subcellular localization of CPB-3 and CGH-1 in PVD neurons, we used total internal reflection fluorescence (TIRF) microscopy (see Materials and Methods). TIRF microscopy uses an excitation laser beam that is totally internally reflected at the glass-water interface and produces an evanescent wave that travels parallel to the interface and selectively excites the sample $100 \mathrm{~nm}-1 \mu \mathrm{m}$ from the substrate. This method greatly reduces background fluorescence and enables tracking of particles within the evanescent field [23].

We imaged a total of 147 CPB-3::GFP particles and 80 CGH-1::GFP particles. For each particle, we calculated the mean-squared displacement (MSD), plotted it as a function of time, and fit the data to the three MSD models described in Equation (Eqs. 1, 2, and 3 to categorize each particle as stationary (measured particle diffusivities below 1.0 e-3 $\mu \mathrm{m}^{2} / \mathrm{s}$ ), diffusive, confined, or as making a directed run (see Materials and Methods). CPB-3 and CGH-1 particle trajectory lengths ranged from 2 to $36 \mathrm{~s}$ with a frame rate $(\Delta t)$ of 50,20 , or 10 frames/s. Estimated particle localization uncertainty for all trajectories was determined by fitting the free, unconfined diffusion MSD Eq. (1) to the first three $2 \Delta t$, $3 \Delta t$, and $4 \Delta t$ time points of the MSD plot. The localization uncertainty was $48 \pm 21 \mathrm{~nm}$ for $\Delta t=20 \mathrm{~ms}$, $37 \pm 23 \mathrm{~nm}$ for $\Delta t=50 \mathrm{~ms}$, and $34 \pm 21 \mathrm{~nm}$ for $\Delta t=$ $100 \mathrm{~ms}$ where the error represents standard error of the mean (SEM). The corresponding average uncertainty in CPB-3 and CGH-1 particle localization was calculated to be $40 \pm 22 \mathrm{~nm}$.

For CPB-3::GFP particles, $8 \%$ were stationary, $36 \%$ were diffusive, $48 \%$ exhibited confined movement, and $8 \%$ of particles made extended runs of directional movement. For CGH-1::GFP particles, $14 \%$ were stationary, 39\% were diffusive, $1 \%$ confined (a single particle), and $46 \%$ made runs (Table 1; Fig. 1). For particles that made directed runs within dendrites, we calculated their median speed at $0.68 \mu \mathrm{m} / \mathrm{s}$ for $\mathrm{CPB}-3$ and $0.87 \mu \mathrm{m} / \mathrm{s}$ for $\mathrm{CGH}-1$ (Table 1 ). There was no statistically significant difference between CPB-3 and CGH-1 particle run speed ( $\mathrm{p}=0.9$ by two-sample $\mathrm{t}$-test).

We also evaluated the direction of CPB-3 and CGH-1 particle movement within dendrites. Of 60 CPB-3::GFP particles exhibiting directed movement, $45 \%(n=27)$ exhibited anterograde movement away from the soma, $18 \%(n=11)$ exhibited retrograde movement, and $37 \%$ $(n=22)$ exhibited bidirectional movement. Of 52 CGH1::GFP particles exhibiting directed movement, $23 \%$ $(n=12)$ were anterograde, $12 \%(n=6)$ were retrograde, and $65 \%(n=34)$ were bidirectional (see Fig. 1$)$. 
Table 1 Parameter vales obtained from MSD fits. SEM represents standard error of the mean

\begin{tabular}{|c|c|c|c|c|c|c|}
\hline $\begin{array}{l}\text { Mode of transport (\# } \\
\text { of cells) }\end{array}$ & Fit Eq & \# of tracks & $\begin{array}{l}\text { Speed }(\mu \mathrm{m} / \mathrm{s}), \\
\text { Median, }\left(75^{\mathrm{th}},\right. \\
\left.25^{\text {th }}\right)\end{array}$ & $\begin{array}{l}\text { Diffusivity }\left(\mu \mathrm{m}^{2} / \mathrm{s}\right) \\
\text { Median, }\left(75^{\text {th }}, 25^{\text {th }}\right)\end{array}$ & $\begin{array}{l}\text { Confinement length } \\
L_{x}(\mu \mathrm{m}), \text { Median, }\left(75^{\text {th }},\right. \\
\left.25^{\text {th }}\right)\end{array}$ & $\begin{array}{l}\text { Confinement length } \\
L_{y}(\mu \mathrm{m}), \text { Median, }\left(75^{\text {th }},\right. \\
\left.25^{\text {th }}\right)\end{array}$ \\
\hline CPB-3 & & 147 & & & & \\
\hline Stationary ( 5 cells) & Equation (1) & 12 & & 3.2 e-4 (5.4 e- $4,1.2$ e-4) & & \\
\hline Diffusive ( 9 cells) & Equation (1) & 53 & & $0.0087(0.052,0.0023)$ & & \\
\hline Confined (10 cells) & Equation (3) & 70 & & $0.087(0.32,0.0085)$ & $0.61(0.99,0.21)$ & $0.037(0.082,0.026)$ \\
\hline Runs (7 cells) & Equation (2) & 12 & $0.68(1.97,0.24)$ & & & \\
\hline $\mathrm{CGH}-1$ & & 80 & & & & \\
\hline Stationary (7 cells) & Equation (1) & 11 & & 4.1 e-4 (7.4 e- $4,2.5$ e-4) & & \\
\hline Diffusive (11 cells) & Equation (1) & 31 & & $0.010(0.027,0.0044)$ & & \\
\hline Confined (1 cell) & Equation (3) & 1 & & 0.29 & 0.46 & 0.39 \\
\hline Runs ( 10 cells) & Equation (2) & 37 & $0.87(1.65,0.43)$ & & & \\
\hline
\end{tabular}

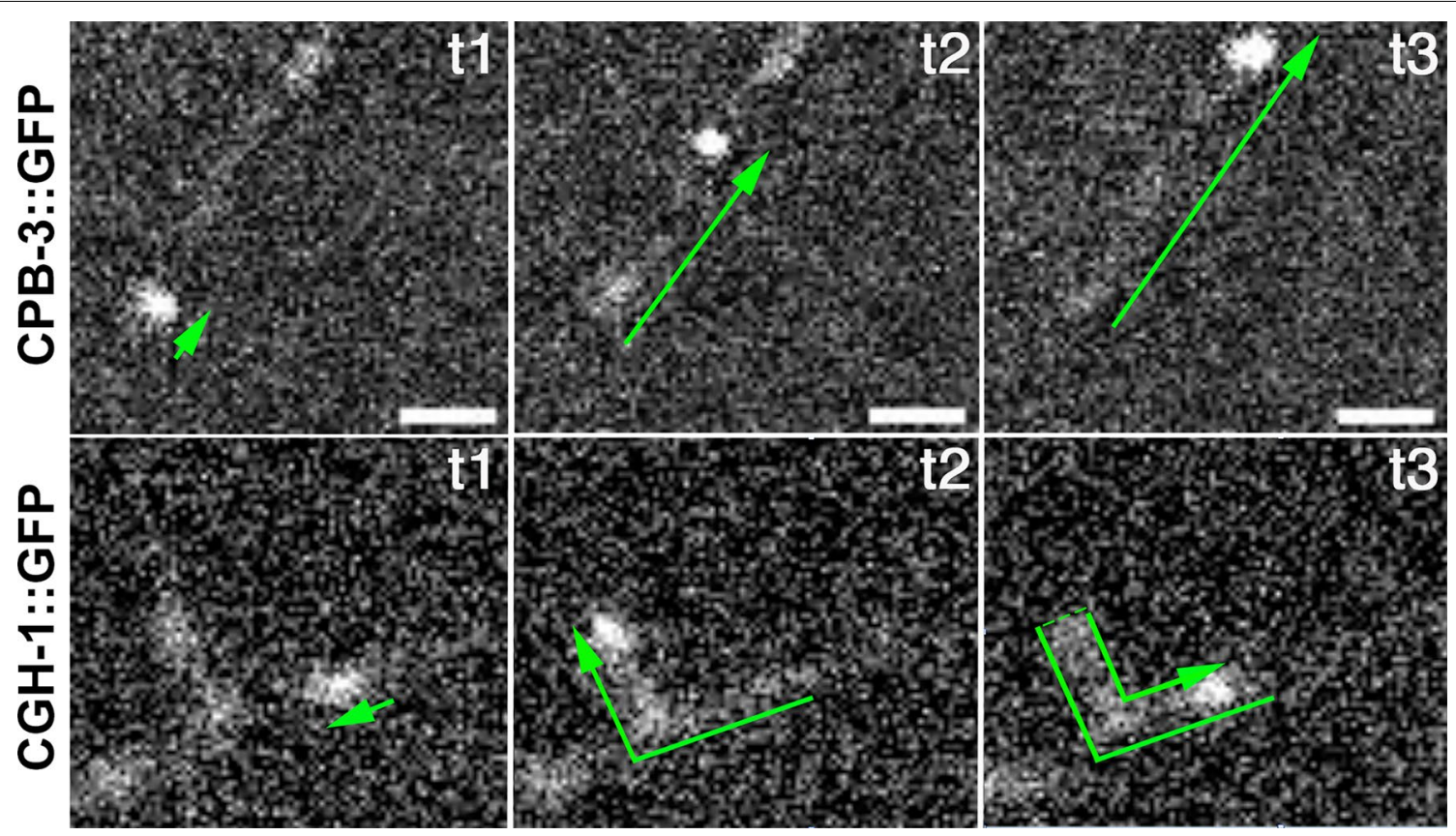

Fig. 1 CPB-3 and CGH-1 particles are motile in PVD dendrites. Arrows outline movement over time where t1, t2, and t3 are different images from a time lapse movie with an acquisition frequency of $100 \mathrm{~ms}$. Note: $\mathrm{CGH}-1$ reverses direction. $\mathrm{Bar}=1 \mu \mathrm{m}$

\section{CPB-3 and CGH-1 mostly localize to distinct cytoplasmic particles}

To learn if CPB-3 and CGH-1 localize to the same particles, we expressed CPB-3::GFP and CGH-1::mCherry fusion proteins in PVD neurons, imaged them using confocal microscopy, and performed a colocalization analysis (see Materials and methods; Fig. 2). We found that CPB-3::GFP and CGH-1::mCherry particles have a Pearson correlation coefficient of 0.168 where 0 represents no colocalization and 1 is perfect colocalization
[24]. Thus, the data suggest, at best, a weak colocalization of CBP-3 and CGH-1 particles. Furthermore, the correlation coefficient may be an overestimate given that CBP-3 and CGH-1 particles could have been trapped within the same compartment, moving around within the same confinement zone, thus appear colocalized due to their proximity and the limitations of confocal microscopy. Nonetheless, the majority of CPB-3 and $\mathrm{CGH}-1$ foci do not colocalize. 

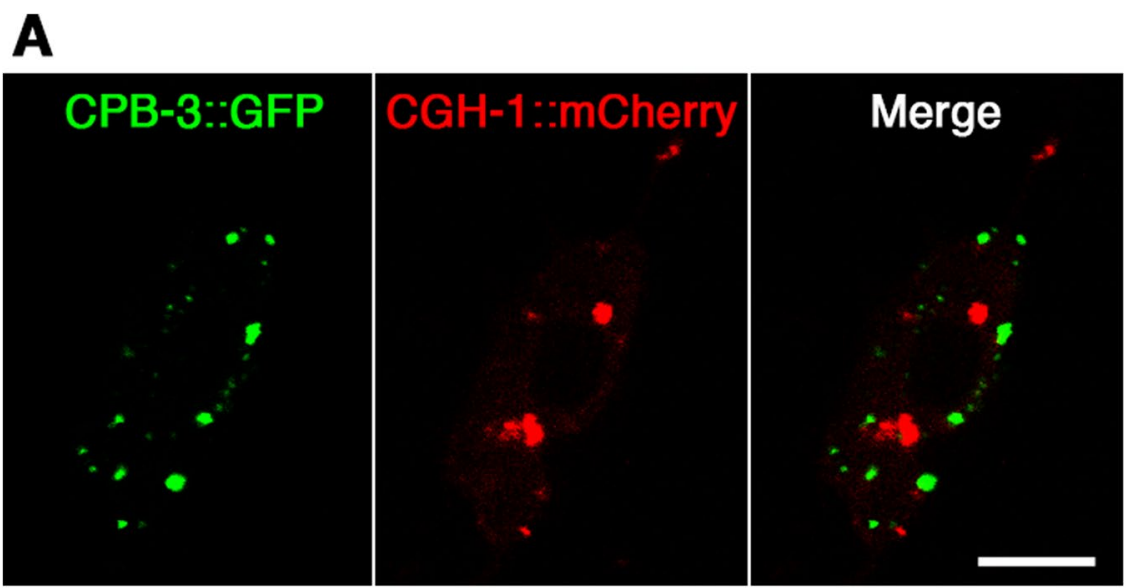

B

Fig. 2 A CPB-3::GFP and CGH-1::mCherry label distinct particles in the cytoplasm of PVD neurons. Bar = $5 \mu$ m. B 58 confocal Z-stack images from 12 PVD neurons were analyzed by for colocalization using the ImageJ plug-in Coloc2 to obtain Pearson's correlation coefficients. The average correlation coefficient is 0.168 ; error bars represent the $95 \%$ confidence interval

\section{Conclusions}

Here we show that $\mathrm{CPB}-3$ and $\mathrm{CGH}-1$ localize to cytoplasmic particles within PVD neurons, some of which are motile within dendrites. This is consistent with CPB-3 and $\mathrm{CGH}-1$ regulating posttranscriptional gene expression by acting within RNPs to regulate mRNA transport, localization, stability, and localized translation. Importantly, CPB-3 and CGH-1 are likely to have evolutionarily conserved roles in regulating RNA within dendrites, as $\mathrm{CPEB}$ is found in motile particles in vertebrate dendrites and Me31B localizes to RNPs within dendrites in Drosophila [13-15, 22]. The underlying architecture of dendrites is based on microtubules and numerous studies have implicated the microtubule-based motor proteins kinesin and dynein in the transport of RNPs within dendrites [25-29]. The speed of particles with directed movement that we observed $(0.68-0.87 \mu \mathrm{m} / \mathrm{s})$ is consistent with observed speeds for kinesin-mediated and dynein-mediated transport within neurons $(\sim 1 \mu \mathrm{m} / \mathrm{s})$ [30]. Importantly, we see CPB-3 and CGH-1 are mostly localized to distinct particles. This suggests that CPB-3 and CGH-1 may regulate different mRNAs and/or function independently of each other. Together, our results support a model in which $\mathrm{CPB}-3$ and $\mathrm{CGH}-1$ regulate mRNAs during their transport within dendrites, perhaps playing a critical role in regulating dendrite morphogenesis.

\section{Materials and Methods \\ C. elegans genetics and transgenic strains}

Strains were derived from the Bristol strain N2, grown at $20 \mathrm{C}$, and constructed using standard procedures
[31]. For analysis of motile particles within PVD dendrites, $c p b-3$ and $c g h-1$ cDNAs were expressed as fusion proteins to GFP under the control of the PVD-specific promoter ser2prom3 [6, 32]. For colocalization studies, strain DJK172: unc-76(e911); cnjEx[s2p3::cpb-3::GFP, s2p3::cgh-1::cherry, unc-76(+)] was created by injecting plasmid pDJK247 (ser2prom3::cpb-3 cDNA::GFP) at $20 \mathrm{ng} / \mathrm{ul}, \mathrm{pDJK} 293$ (ser2prom3::cgh-1 cDNA::mCherry) at $20 \mathrm{ng} / \mathrm{ul}$, and $u n c-76(+)$ at $60 \mathrm{ng} / \mathrm{ul}$ into unc76(e911) animals. DNA microinjection was performed using standard practices [33]. For all microscopy, adult animals were mounted on coverslips with $2 \%$ agarose pads, and immobilized with $600 \mathrm{mM}$ levamisole.

\section{TIRF microscopy}

TIRF microscopy was used to visualize CPB-3::GFP and CGH-1::GFP motion. The penetration depth of the evanescent wave for $488 \mathrm{~nm}$ excitation was between 600-700 nm. Objective-based TIRF microscopy was performed with a S-TIRF module (Spectral Applied Research, Canada) attached to a Leica DMI3000 B inverted microscope with a $100 \times$ and 1.47 N.A. oil immersion objective using a $488 \mathrm{~nm}$ laser (Coherent Inc.). A $1.5 \times$ lens was added to the excitation beam path resulting in a final magnification of $150 x$. A $550 / 20 \mathrm{~nm}$ single-band bandpass filter (Chroma) was used to collect fluorescence. TIRF images were collected at room temperature with an EMCCD camera (Evolve Delta; Photometrics) operated by Micro-Manager [34]. Image series were collected with image exposure of $20 \mathrm{~ms}, 50 \mathrm{~ms}$ or $100 \mathrm{~ms}$ depending on particle brightness. 


\section{Single-particle tracking}

Images were processed in MATLAB (MathWorks, Inc., Natick, MA), in conjunction with the DIPImage image processing toolbox [35]. Image backgrounds were averaged and subtracted to reduce noise. Particle coordinates $(\mathrm{x}, \mathrm{y})$ were identified in each frame by a direct Gaussian fit algorithm [36, 37] and linked together into trajectories. To identify the nature of particle motion, the $\operatorname{MSD}\left\langle r^{2}(\Delta t)\right\rangle$, a measure of the average (denoted by brackets) distance a molecule travels, was calculated for each time difference $\Delta t$ in the track record. After computing the MSD, a plot of square-displacements as a function of time has the ability to resolve different modes of particle motion [38]. The MSD signature for two-dimensional free, unconfined diffusion is linear.

$$
\left\langle r^{2}(\Delta t)\right\rangle=\text { offset }+4 D \Delta t,
$$

where $D$ is the particle's diffusion coefficient and the offset represents the sum of the localization error [39]. For directed particle motion (runs) with speed $v$, the MSD plot exhibits an upward curvature.

$$
\left\langle r^{2}(\Delta t)\right\rangle=\text { offset }+4 D \Delta t+v^{2} \Delta t^{2} .
$$

The third observed two-dimensional mode of particle motion, was confined diffusion with MSD described as.

$$
\begin{aligned}
\left\langle r^{2}(\Delta t)\right\rangle= & \text { offset }+\frac{L_{x}^{2}}{6}\left[1-\exp \left(-\pi^{2} D \Delta t / L_{x}^{2}\right)\right] \\
& +\frac{L_{y}^{2}}{6}\left[1-\exp \left(-\pi^{2} D \Delta t / L_{y}^{2}\right)\right] .
\end{aligned}
$$

In confined diffusion the MSD graph has a downward curvature and asymptotically approaches $\mathrm{L}_{\mathrm{x}}{ }^{2} / 6$ and $\mathrm{L}_{\mathrm{y}}{ }^{2} / 6$. In this mode the particle diffuses within a limited area bounded by $0 \leq \mathrm{x} \leq \mathrm{L}_{\mathrm{x}}$ and $0 \leq \mathrm{y} \leq \mathrm{L}_{\mathrm{y}}$.

\section{Directionality of CPB-3 and CGH-1 particles}

Observation of particle direction was performed with a Leica DM500B epifluorescence microscope with a $63 \times$ objective.

\section{Colocalization of CPB-3 and CGH-1}

Strain DJK247 was imaged with a Leica SP5 spectral confocal microscope at $63 \times$ with $0.5 \mu \mathrm{m}$ per step and Leica LAS software. Colocalization of CPB-3::GFP and CGH1::Cherry in PVD neurons was analyzed by ImageJ plug-in Coloc2 (http://fiji.sc/Coloc2) on regions of interest corresponding to the PVD soma to obtain Pearson's correlation coefficients $[10,40]$.

\section{Limitations of the study}

- To facilitate the visualization of RBP-containing particles we used a transgene expression system that likely leads to overexpression. We cannot rule out the possibility that overexpression may impact the number, size, and behavior of the RBP-containing particles.

- While we used a highly sensitive TIRF microscopy system to image RBP-containing particles, we cannot rule out the possibility that some particles were below the threshold for our methods of detection.

- Since this study was restricted to examining particles within PVD neurons in C. elegans, it is not clear if CPB-3 and CGH-1 localize to motile particles with similar properties in other types of neurons, or if their orthologs do so in other species.

\section{Abbreviations}

RBP: RNA-binding protein; C. elegans: Caenorhabditis elegans; CPEB: Cytoplasmic polyadenylation element binding protein; RNP: Ribonucleoprotein particle; da neuron: Dendrite arborization neuron; P-body: Processing body; TIRF: Total internal reflection microscopy; MSD: Mean-squared displacement; Eq: Equation; SEM: Standard error of the mean; GFP: Green fluorescent protein.

\section{Acknowledgements}

We thank Delaine Winkelblech and Brent Wallace for technical support.

\section{Authors' contributions}

DJK and MW constructed the transgenic strains. EOC performed confocal microscopy and MW and DF performed the analyses. KS performed the TIRF microscopy and subsequent analyses. EOC, KS, and DJK wrote and edited the manuscript and constructed the tables and figures. All authors read and approved the final manuscript.

\section{Funding}

This work was supported by the Directorate for Biological Science-National Science Foundation (IOS Proposal Number 1257703 to DJK and 1257656 to $\mathrm{ECO}$.

Availability of data and materials

C. elegans strains and transgenes (plasmids) are available upon request.

\section{Declarations}

Ethics approval and consent to participate Not applicable.

\section{Consent for publication}

Not applicable.

\section{Competing interests}

The authors have no competing interests.

Received: 21 May 2021 Accepted: 4 August 2021 Published online: 14 August 2021 


\section{References}

1. Jan YN, Jan LY. Branching out: mechanisms of dendritic arborization. Nat Rev Neurosci. 2010;11:316-28.

2. Kulkarni VA, Firestein BL. The dendritic tree and brain disorders. Mol Cell Neurosci. 2012;50:10-20. https://doi.org/10.1016/j.mcn.2012.03.005.

3. Holt CE, Schuman EM. The central dogma decentralized: new perspectives on RNA function and local translation in neurons. Neuron. 2013;80:648-57. https://doi.org/10.1016/J.NEURON.2013.10.036.

4. Khalil B, Morderer D, Price PL, Liu F, Rossoll W. mRNP assembly, axonal transport, and local translation in neurodegenerative diseases. Brain Res. 2018;1693:75-91. https://doi.org/10.1016/J.BRAINRES.2018.02.018.

5. Olesnicky EC, Killian DJ, Garcia E, Morton MC, Rathjen AR, Sola IE, et al. Extensive use of RNA-binding proteins in drosophila sensory neuron dendrite morphogenesis. G3 Genes Genomes Genet. 2014:4:1.

6. Antonacci S, Forand D, Wolf M, Tyus C, Barney J, Kellogg L, et al. Conserved RNA-binding proteins required for dendrite morphogenesis in Caenorhabditis elegans sensory neurons. G3 Genes Genomes Genet. 2015;5:1.

7. Olesnicky EC, Killian DJ. The cytoplasmic polyadenylation element binding protein (CPEB ), Orb, is important for dendrite development and neuron fate specification in Drosophila melanogaster. Gene. 2020;738:144473. https://doi.org/10.1016/j.gene.2020.144473.

8. Schachtner LT, Sola IE, Forand D, Antonacci S, Postovit AJ, Mortimer NT, et al. Drosophila Shep and C. elegans SUP-26 are RNA-binding proteins that play diverse roles in nervous system development. Dev Genes Evol. 2015;225:319-30.

9. Olesnicky EC, Bono JM, Bell L, Schachtner LT, Lybecker MC. The RNAbinding protein caper is required for sensory neuron development in Drosophila melanogaster. Dev Dyn. 2017;246:610-24.

10. Olesnicky EC, Antonacci S, Popitsch N, Lybecker MC, Titus MB, Valadez R, et al. Shep interacts with posttranscriptional regulators to control dendrite morphogenesis in sensory neurons. Dev Biol. 2018;444(2):116-28.

11. Richter JD. CPEB: a life in translation. Trends Biochem Sci. 2007;32:279-85. https://doi.org/10.1016/J.TIBS.2007.04.004.

12. Ivshina M, Lasko P, Richter JD. Cytoplasmic polyadenylation element binding proteins in development, health, and disease. Annu Rev Cell Dev Biol. 2014;30:393-415. https://doi.org/10.1146/annurev-cellb io-101011-155831.

13. Huang YS, Carson JH, Barbarese E, Richter JD. Facilitation of dendritic mRNA transport by CPEB. Genes Dev. 2003;17:638-53.

14. Bestman JE, Cline HT. The RNA binding protein CPEB regulates dendrite morphogenesis and neuronal circuit assembly in vivo. Proc Natl Acad Sci. 2008;105:20494-9. https://doi.org/10.1073/pnas.0806296105.

15. Bestman JE. The relationship between dendritic branch dynamics and CPEB-labeled RNP granules captured in vivo. Front Neural Circuits. 2009. https://doi.org/10.3389/neuro.04.010.2009.

16. Barbee SA, Estes PS, Cziko AM, Hillebrand J, Luedeman RA, Coller JM, et al. Staufen- and FMRP-containing neuronal RNPs are structurally and functionally related to somatic p bodies. Neuron. 2006;52:997-1009.

17. Tang NH, Kim KW, Xu S, Blazie SM, Yee BA, Yeo GW, et al. The mRNA decay factor CAR-1/LSM14 regulates axon regeneration via mitochondrial calcium dynamics. Curr Biol. 2020;30(5):865-76.

18. De Valoir T, Tucker MA, Belikoff EJ, Camp LA, Bolduc C, Beckingham K. A second maternally expressed Drosophila gene encodes a putative RNA helicase of the "DEAD box" family. Proc Natl Acad Sci USA. 1991;88(6):2113-7.

19. Navarro RE, Shim EY, Kohara Y, Singson A, Blackwell TK. cgh-1, a conserved predicted RNA helicase required for gametogenesis and protection from physiological germline apoptosis in C. elegans. Development. 2001;128(17):3221-32

20. Presnyak V, Coller J. The DHH1/RCKp54 family of helicases: An ancient family of proteins that promote translational silencing. Biochimica et Biophysica Acta - Gene Regulatory Mechanisms. 2013;1829(8):817-23.
21. Weston A, Sommerville J. Xp54 and related (DDX6-like) RNA helicases: Roles in messenger RNP assembly, translation regulation and RNA degradation. Nucleic Acids Res. 2006;34(10):3082-94.

22. Hillebrand J, Pan K, Kokaram A, Barbee S, Parker R, Ramaswami M. The Me31B DEAD-box helicase localizes to postsynaptic foci and regulates expression of a CaMKII Reporter mRNA in dendrites of drosophila olfactory projection neurons. Front Neural Circuits. 2010. https://doi.org/10. 3389/fncir.2010.00121.

23. Martin-Fernandez ML, Tynan CJ, Webb SED. A "pocket quide" to total internal reflection fluorescence. J Microsc. 2013;252(1):16-22.

24. Dunn KW, Kamocka MM, McDonald JH. A practical guide to evaluating colocalization in biological microscopy. Am J Physiol. 2011:300(4):C723-42.

25. Bramham CR, Wells DG. Dendritic mRNA: Transport, translation and function. Nature Reviews Neuroscience. 2007;8(10):776-89.

26. Hirokawa N. mRNA transport in dendrites: RNA Granules, Motors, and Tracks. J Neurosci. 2006;26:7139-42. https://doi.org/10.1523/JNEUROSCI. 1821-06.2006.

27. Kapitein LC, Hoogenraad CC. Which way to go? Cytoskeletal organization and polarized transport in neurons. Mol Cell Neurosci. 2011;46:9-20. https://doi.org/10.1016/j.mcn.2010.08.015.

28. Xu X, Brechbiel JL, Gavis ER. Dynein-dependent transport of nanos RNA in Drosophila sensory neurons requires Rumpelstiltskin and the germ plasm organizer Oskar. J Neurosci. 2013;33:14791-800. https://doi.org/10.1523/ JNEUROSCI.5864-12.2013.

29. De Graeve F, Bessé F. Neuronal RNP granules: From physiological to pathological assemblies. Biol Chem. 2018;399(7):623-35.

30. Maday S, Twelvetrees AE, Moughamian AJ, Holzbaur ELF. Axonal Transport: Cargo-Specific Mechanisms of Motility and Regulation. Neuron. 2014:84(2):292-309.

31. Brenner S. The genetics of Caenorhabditis elegans. Genetics. 1974;77:95-104.

32. Liu OW, Shen K. The transmembrane LRR protein DMA-1 promotes dendrite branching and growth in C. elegans. Nat Neurosci. 2012;15:57-63. https://doi.org/10.1038/nn.2978.

33. Mello C, Fire A. DNA Transformation. Methods Cell Biol. 1995;48:451-82

34. Edelstein A, Amodaj N, Hoover K, Vale R, Stuurman N. Computer Control of Microscopes Using $\mu$ Manager. In: Current Protocols in Molecular Biology. Hoboken: Wiley; 2010. pp 14.20.1-14.20.17.

35. Hendriks CLL, van Vliet LJ, Rieger B, van Kempen GMP, van Ginkel M. Dipimage: a scientific image processing toolbox for MATLAB. Quantitative Imaging Group: Faculty of Applied Sciences, Delft University of Technology, Delft, The Netherlands; 1999.

36. Andrews NL, Lidke KA, Pfeiffer JR, Burns AR, Wilson BS, Oliver JM, et al. Actin restricts $F(\varepsilon R I$ diffusion and facilitates antigen-induced receptor immobilization. Nat Cell Biol. 2008;10:955-63.

37. Spendier K, Lidke KA, Lidke DS, Thomas JL. Single-particle tracking of immunoglobulin e receptors (FCRI) in micron-sized clusters and receptor patches. FEBS Lett. 2012;586:1.

38. Kusumi A, Sako Y, Yamamoto M. Confined lateral diffusion of membrane receptors. Biophys J. 1993;65(5):2021-40.

39. Savin T, Doyle PS. Static and dynamic errors in particle tracking microrheology. Biophys J. 2005:88:623-38.

40. Chen Y, Bharill S, Altun Z, O'Hagan R, Coblitz B, Isacoff EY, et al. Caenorhabditis elegans paraoxonase-like proteins control the functional expression of DEG/ENaC mechanosensory proteins. Mol Biol Cell. 2016;27(8):1272-85.

\section{Publisher's Note}

Springer Nature remains neutral with regard to jurisdictional claims in published maps and institutional affiliations. 